\title{
Imaging in resectable colorectal liver metastasis patients with or without preoperative chemotherapy: results of the PROMETEO-01 study
}

\author{
F L Rojas Llimpe ${ }^{1}$, F Di Fabio ${ }^{1}$, G Ercolani ${ }^{2}$, E Giampalma ${ }^{3}$, A Cappelli ${ }^{3}$, C Serra ${ }^{4}$, P Castellucci ${ }^{5}$, A D'Errico ${ }^{6}$, \\ R Golfieri ${ }^{3}$, A D Pinna ${ }^{2}$ and C Pinto*,1 \\ ${ }^{1}$ Medical Oncology Unit, S. Orsola-Malpighi Hospital, Bologna 40138, Italy; ${ }^{2}$ Liver Surgery Unit, S. Orsola-Malpighi Hospital, \\ Bologna 40138, Italy; ${ }^{3}$ Radiology Unit, S. Orsola-Malpighi Hospital, Bologna 40138, Italy; ${ }^{4}$ Internal Medicine Unit, S. Orsola-Malpighi \\ Hospital, Bologna 40138, Italy; ${ }^{5}$ Nuclear Medicine Unit, S. Orsola-Malpighi Hospital, Bologna 40138, Italy and ${ }^{6}$ Pathology Unit, \\ S. Orsola-Malpighi Hospital, Bologna 40138, Italy
}

Background: The aim of the PROMETEO-01 Study was to define the diagnostic accuracy of imaging techniques in colorectal cancer liver metastasis (CRCLM) patients.

Methods: Patients referred to Bologna S. Orsola-Malpighi Hospital performed a computed-tomography scan (CT), magnetic resonance (MR), 18F-FDG-PET/CTscan (PET/CT) and liver contrast-enhanced-ultrasound (CEUS); CEUS was also performed intraoperatively (i-CEUS). Every pathological lesion was compared with imaging data.

Results: From December 2007 to August 2010, 84 patients were enrolled. A total of 51 (60.71\%) resected patients were eligible for analysis. In the lesion-by-lesion analysis 175 resected lesions were evaluated: 67(38.3\%) belonged to upfront resected patients (group-A) and 108 (61.7\%) to chemotherapy-pretreated patients (group-B). In all patients the sensitivity of MR proved better than CT (91\% vs 82\%; $P=0.002)$, CEUS (91 vs $81 \% ; P=0.008$ ) and PET/CT (91\% vs $60 \% ; P=0.000$ ), whereas PET/CT showed the lowest sensitivity. In group-A the sensitivity of i-CEUS, MR, CT, CEUS and PET/CT was $98 \%, 94 \%, 91 \%, 84 \%$ and $78 \%$, respectively. In group-B the i-CEUS proved equivalent in sensitivity to MR $(95 \%$ and $90 \%$, respectively, $P=0.227)$ and both were significantly more sensitive than other procedures. The CT sensitivity in group-B was lower than in group-A $(77 \%$ vs $91 \%, P=0.024)$.

Conclusions: A thoraco-abdominal CT provides an adequate baseline evaluation and guides judgment as to the resectability of CRCLM patients. In the subset of candidates for induction chemotherapy to increase the chance of liver resection, the most rational approach is to add MR for the staging and restaging of CRCLM.

Liver metastases (LMs) in colorectal cancer (CRC) are already present at the time of diagnosis of primary tumour in $\sim 15-20 \%$ of cases, whereas $60 \%$ of patients who develop metastatic disease present LM (Wagner et al, 1984). In these patients, surgical resection of metastases resectable $a b$ initio or after preoperative chemotherapy remains the only treatment with potentially curative intent, achieving a 5-year survival rate of 30 and $15 \%$ at 10 years (Ruers and Bleichrodt, 2002; Morris et al, 2010; Vigano et al, 2012; Sorbye et al, 2012).

Developments to surgical techniques and liver resectability criteria, as seen in the last decade, have led us to consider liver surgery as a key step in the therapeutic strategy of patients with 
colorectal cancer liver metastasis (CRCLM; Muratore et al, 2010; Isoniemi and Osterlund, 2011; Lam et al, 2012).This emphasizes the relevance of accurate staging of patients with CRCLM so as to allow identification of the extrahepatic disease, as well as a correct topography of the intrahepatic disease, particularly in patients who have received prior chemotherapy (Van Erkel et al, 2002; Fernandez et al, 2004; Rappeport et al, 2007). Moreover, if in addition we consider the continuous innovations in radiological imaging techniques (RITs) and the availability of new more effective chemotherapy regimens, it seems clear that we need multidisciplinary management of patients with LM, devising the best therapeutic (Van Cutsem et al, 2006; Abdel-Misih et al, 2009; Kemeny, 2010; Masi et al, 2011; Nathan et al, 2012).

One important issue is the absence from current clinical practice of any diagnostic algorithm universally shared for the assessment of patients with CRCLM who might benefit from surgery. A shared strategic vision might, on the other hand, allow more rational use of increasingly limited financial resources. In 2006, at S. OrsolaMalpighi Hospital in Bologna, Italy, a MDT was formed involving various different specialists (hepatobiliary surgeon, radiologist, oncologist, internist, nuclear medicine physicians and pathologist) who meet every week to discuss and assess all cases of patients accessing the hospital with LM from CRC or from other solid tumours. Inside this context, the Bologna' MDT designed a prospective PROMETEO trial, which aimed to assess the various available RITs in patients with CRCLM in order to determine diagnostic accuracy in the assessment of patients undergoing surgical resection and the identification of prognostic biomarkers.

The first objective of the PROMETEO-01 Study was to evaluate the role of computed-tomography scan (CT), magnetic resonance (MR), PET/CT, liver contrast-enhanced-ultrasound (CEUS) and intraoperative CEUS (i-CEUS) in presurgical assessment of patients in order to identify one or two highly sensitive RIT, which in clinical practice may guide patient selection for the planning of surgery. The assessment of each case was prospectively performed by our MDT and recorded in a shared database. We later performed a "lesion-by-lesion" analysis taking as our reference standard the histological examination of every single lesion surgically excised.

\section{PATIENTS AND METHODS}

Patients. Patients presenting to our MDT were evaluated if the carriers of hepatic lesions are morphologically suspected as denoting CRCLM. The suspicious LMs were diagnosed by a CT in 60 out of 84 patients and by liver ultrasound and in 44 out of 84 patients.

Main inclusion criteria: The main exclusion criteria were as follows: histological diagnosis of colorectal adenocarcinoma with lesions to the liver, raising suspicion of synchronous or metachronous metastasis that might potentially be resectable after adequate staging and/or conversion chemotherapy; absence of extrahepatic disease outside the regional lymph nodes and/or the hepatic hilum; and signed written informed consent.

Exclusion criteria: Exclusion criteria were as follows: contraindications against MR, when at least three other examinations could not be performed before the scheduled surgery; and other malignancies.

In 3 weeks before liver surgery, the patients underwent a thoraco-abdominal CT, a liver MR, a total-body PET/CT and CEUS. During the course of surgery was performed an i-CEUS. Patients in group-B who performed the same exams three weeks after the latest administration of chemotherapy. Patients who were considered not easily resectable after being evaluated by three independent surgeons, as part of the MDT, underwent chemotherapy (Quan et al, 2012).

\section{Radiological Imaging Techniques.}

Multidetector Computed Tomography (MDCT): The CT study was performed using a 6-slice MDCT scanner (Sensation 6 Siemens, Erlangen, Germany). Scan was obtained from the diaphragm to the pubic symphysis in the pre-contrast, arterial and late phases, including also the chest during the venousportal acquisition.

Scans were acquired using the following parameters: 5-mm slice thickness, pitch $5.5,130 \mathrm{kV}, 130 \mathrm{mAs}$ and $181 \mathrm{~mA}$. Images were reconstructed with a $2.5-\mathrm{mm}$-effective thickness and using a $3 \mathrm{D}$ Multi-Planar Reformatting and/or Maximum Intensity Projection when it was necessary to better identify the relation with vascular structures and thus the resectability.

The procedure was performed at baseline and after the administration of 150-180 cc of tri-iodinated non-ionic contrast agent, IomeronÒ Bracco, Milan, Italy $\left(350 \mathrm{mgI} \mathrm{ml}^{-1}\right)$ at a flow rate of $3-4 \mathrm{ml} \mathrm{s}^{-1}$ into an anticubital vein by means of an 18-gauge needle using an automated power injection.

The examination was perfomed using the multiphasic technique and bolus tracking: a region of interest was placed over the abdominal aorta at the level of the celiac tripod and a threshold of $150 \mathrm{HU}$ (Hounsfield Unit) was selected. Once the threshold had been reached, three scans were performed with delays of 20-25 s (arterial phase), 45-60s (portalvenous phase) and 180-300 s (delayed phase).

All images were transferred to a workstation and evaluated using the institutional Picture Archiving and Communication System (PACS; Carestream PACS, version 1.1, Kodak, Rochester, NY, USA), and the radiological reports stored in the Radiological Informative System (Exprivia Project Spa, version e-ris, Rome, Italy).

Magnetic resonance: Magnetic resonance was performed with a 1.5-T superconducting system (Signa, GE Medical Systems, Milwaukee, WI, USA), using a body-phased array of multicoils for signal detection at baseline and after intravenous injection of Gd-EOB-DTPA (PrimovistÒ, Bayer Schering Pharma AG, Berlin, Germany) at a dose of $0.1 \mathrm{ml} \mathrm{kg}^{-1}$ body weight (injection volume of 9-14 $\mathrm{ml}$ according to body weight). Images were acquired in the axial plane, with a section thickness of $4 \mathrm{~mm}$, an intersection gap of $0 \mathrm{~mm}$ and a $28-$ to $35-\mathrm{cm}$ field of view, with the exception of a 3D breath-hold fast spoiled GRE (gradient-echo) sequence obtained after gadolinium-EOB-DTPA injection, acquired with a slice thickness of $4-5 \mathrm{~mm}$, with an effective section thickness of 2-2.5 mm. Scanning delay times were determined using real-time MRI fluoroscopic monitoring after contrast administration. Both dynamic and $\mathrm{HB}$ phase images were obtained using a fat-suppressed 3D GRE sequence (Liver Acquisition with Volume Acceleration: LAVA, GE Medical Systems) before and after intravenous bolus administration of Gd-EOB-DTPA.

PET/CT: The PET/CT was performed with GE, Discovery LS or GE Discovery STE. PET images were acquired in 2D mode for $4 \mathrm{~min}$ per bed position or $3 \mathrm{D}$ for $2 \mathrm{~min}$ per bed position and reconstructed using OSEM.

All the patients fasted for at least $6 \mathrm{~h}$, maintaining blood glucose levels below $160 \mathrm{mg} \mathrm{ml}^{-1}$, before intravenous injection of $370-555 \mathrm{MBq}$ of FDG $\left(5.5 \mathrm{MBq} \mathrm{kg}^{-1}\right)$. The PET/CT started $60 \mathrm{~min}$ after this injection. The parameters of the helical multidetector CT scan were $120 \mathrm{kV}, 80 \mathrm{~mA}, 0.8 \mathrm{~s}$ per tube rotation, slice thickness $3.75 \mathrm{~mm}$, pitch $1.675: 1$ and table speed $33.5 \mathrm{~mm}$ per rot. CT images were used for both attenuation correction of emission data and image fusion. No oral or intravenous contrast media were administered for the CT portion. Metabolic and morphologic studies were evaluated with 
dedicated software (Xeleris; GE Healthcare), which allowed us to review $\mathrm{PET}, \mathrm{CT}$ and fusion images.

CEUS and i-CEUS: Ultrasound (US) and CEUS examinations were performed using iU22 equipment (Philips Medical Systems, Bothel, WA, USA) with a convex $2-5 \mathrm{mHz}$ probe and, when necessary, a linear $3-9 \mathrm{mHz}$ probe.

In the pre-operatory evaluation, all examinations started with B-mode evaluation; subsequently, $2.4 \mathrm{ml}$ SonoVue (Bracco, Milan, Italy) were injected into the antecubital vein. This was repeated if necessary.

During the intra-operatory evaluation, at first, i-US was carried out to search for new nodules (Torzilli, 2004). Second, i-CEUS was carried out both for lesion characterization and for new nodule detection. At the time of the study there were no dedicated probes built to carry out CEUS in direct contact with the targeted organ; therefore, we used the $2-5 \mathrm{mHz}$ convex probe and the $3-9 \mathrm{mHz}$ linear probe. If new lesions were detected in B-mode, i-CEUS was performed in arterial, venous and late phases to distinguish between benign and malignant lesions. If no new lesions were detected in B-mode the whole liver was scanned after $90 \mathrm{~s}$, looking for hypovascular areas that were considered as CRLM.

RIT analysis and MDT. For CT and MR, two radiologists with at least 10 years' experience, not involved in the scanning and not aware of patients' clinical history, read independently the images. In cases of disagreement, there was a third blinded observed who made the final decision. The two observers assessed the lesions for: (1) site according to the Couinaud Classification (Couinaud, 1957); (2) size (bi-diameters); (3) baseline density relative to the surrounding parenchima (iso, hypo or hyperdense/intense); (4) enhancement pattern during the dynamic study (iso, hypo or hyperdense/intense lesions). Enhancement was also assessed in terms of homogeneity (diffuse, filling the lesions with equal intensity) or heterogeneity (rim-like, globular, centripetal and centrifugal). Finally, the readers were asked to provide a diagnosis of the nature of the lesions (metastasis or other benign/malignant lesions).

CT: Metastasis was defined as a lesion iso or hypodense in the pre-contrast phase. Relatively rarely metastasis can reveal a hyperdense appearance because of intralesional bleeding phenomena (more commonly seen in large lesions) or shows intralesional calcifications because of previous chemotherapy cycles. During the post-contrast study, metastasis was defined as a lesion hypoperfused or with an irregular peripheral uptake ('target sign') during the arterial phase and hypovascular (hypodense pattern) during the portal-venous and/or late phase.

$M R$ : Metastasis was defined as a lesion moderately hypointense on T1-weighted images and hyperintense on T2-weighted images or isointense in both sequences. During the dynamic phases, metastasis was defined as hypoperfused or with irregular peripheral enhancement ('target sign') during the arterial phase and hypointense during the portal-venous and/or hepatobiliary phase.

PET: The PET/CT images were revised by two experienced nuclear medicine physicians unaware of the clinical data and the diagnosis was reached by consensus.

CEUS: CEUS was performed by an internist blinded to the results of other RIT. Intraoperative CEUS was clearly not blinded and therefore its sensitivity is overestimated.

The images and radiological reports were then assessed by our MDT who systematically re-discussed and planned the therapeutic strategy for each patient: patients having hepatic lesions with characteristics of malignancy were sent for surgery directly or after chemotherapy treatment, whereas those having isolated hepatic lesions with radiological characteristics of benignity were not resected. At the end of each meeting, data were recorded prospectively on a shared database.
Evaluation criteria. MDT chose the histological examination of each lesion removed as reference standard; hence, lesions that certainly appeared benign by RIT were not resected and were accordingly excluded from the final analysis.

The following step consisted of a multidisciplinary review of images, radiology reports, surgical reports and histopathological reports of all liver lesions resected where a consensus decision was taken whether each liver lesion, suspect or certainly metastatic, described in the radiological reports corresponded to the lesion found at the time of surgery and removed for subsequent histological definition. As the reference standard was here the surgical specimen, the MDT chose to exclude from the final analysis lesions with patently benign behaviour that had not been removed. Thus, in our final analysis we had a very small number of true-negative lesions, with a consequent bias in the specificity calculation.

Statistical analysis. The sensitivity, specificity, accuracy, positive predictive value and negative predictive value were calculated by use of standard statistical formulae.

True-positive was any radiologically suspected malignant lesion, confirmed at histological examination. False-positive was any radiologically suspected malignant lesion, resulting as benign at histological examination. False-negative was lesion identified intraoperatively and/or recognised as malignant at histological examination. True-negatives were hepatic benign lesions that had to be resected because of their proximity to malignant lesions, yet whose benignity was confirmed by histological examination. As a rational decision was taken not to resect all benign liver lesions if not needed, the true-negatives in this series are underestimated.

In order to verify any differences in the detection of LM capability among the five RITs, we used the nonparametric Cochran Q-test. Whenever the Cochran Q-test found statistical significance, the McNemar test was applied so as to test the statistical significance of differences in performance between techniques pairwise. For the comparison between independent groups, the $\chi^{2}$-test was used.

All tests were two-sided and were carried out to a $5 \%$ level of significance, using software SPSS for Windows, version 9.0 (SPSS, Chicago, IL, USA).

\section{RESULTS}

This report focuses on analysis of the five RITs as compared with histological examination (lesion-by-lesion analysis) in CRCLM patients who underwent liver surgery, having preoperatively undergone at least three examinations. The role of PET/CT in detecting extrahepatic disease was also explored.

From December 2007 to August 2010, 84 patients with CRCLM were enrolled in the Prometeo study. Twenty-five (29.76\%) patients were not considered resectable because of not resectable liver disease (six), extrahepatic disease (ten), certainly benign liver lesions upon imaging revision (five), complete response after chemotherapy (one), progression disease after chemotherapy (one) and withdrawal of consent (two). Fifty-nine (70.24\%) patients underwent surgery but another eight patients were excluded from the final analysis because of simply exploratory laparotomy (four), examination via less than two radiological techniques (three), whereas one patient with a suspect primary liver tumour was excluded after histopathological confirmation of cholangiocarcinoma by agreement among all members of the MDT.

A total of $51(60.71 \%)$ resected patients were eligible for analysis (Table 1).

In the lesion-by-lesion analysis, a total of 175 resected lesions were evaluated; 67 (38.3\%) belonged to patients resected upfront 


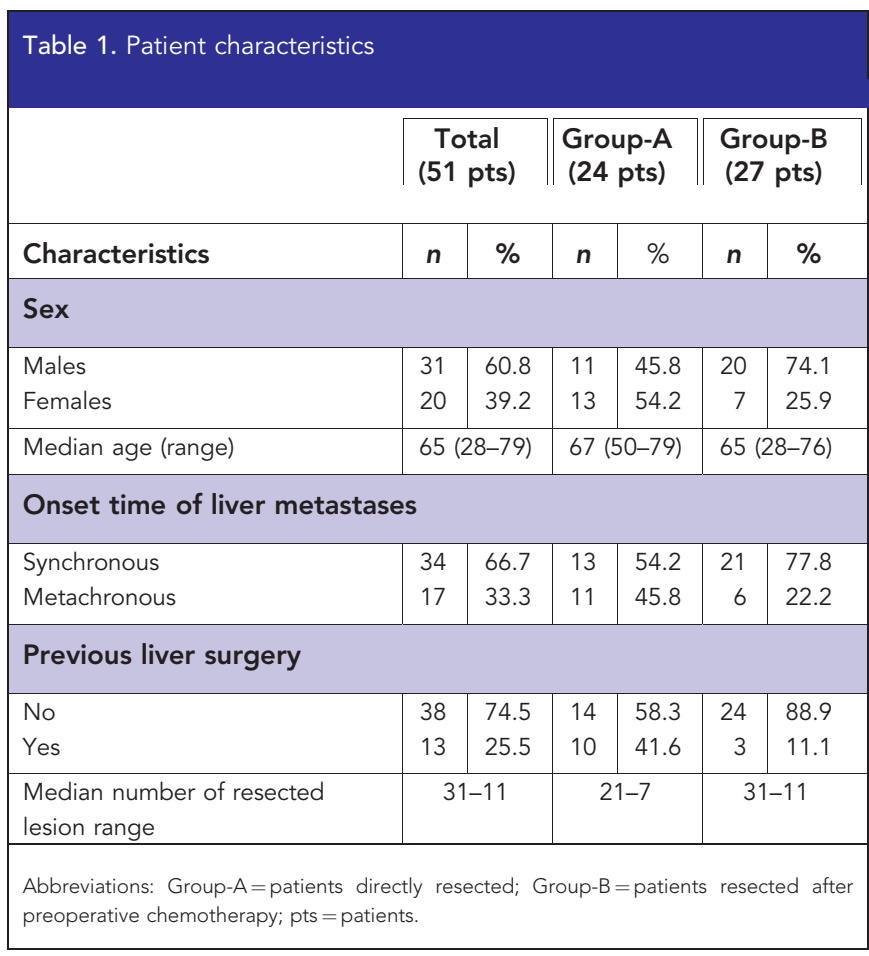

and $108(61.7 \%)$ to chemotherapy-pretreated patients, here named group-A and group- $\mathrm{B}$, respectively.

As reported in Table 2, 155 (88.6\%) lesions were pathologically confirmed as CLRCLM (59 in group-A and 96 in group-B), the other 20 (11.4\%) were classified as: hamartoma (one), cyst (four), necrosis (seven), giant-cell reaction (three), focal nodular hyperplasia (one) and steatosis (four).

The results of lesion-by-lesion analysis of sensitivity, specificity and accuracy of each RIT are reported in Table 3, globally and separately for lesions belong to group-A vs group-B patients.

The five RITs showed statistically different sensitivity in detecting CLRLM (Cochran's Q 100.807, $P=0.000$ ). The statistical significance of differences in performance by each method as compared with another was evaluated by the McNemar test. i-CEUS showed the highest sensitivity that was not statistically significantly different from MR ( $96 \%$ vs $91 \%$; $P=0.092)$. MR was statistically more sensitive than CT $(91 \% v s$ $82 \% ; P=0.002)$, CEUS (91\% vs $81 \% ; P=0.008)$ and $\mathrm{PET} / \mathrm{CT}$ (91\% vs $60 \% ; P=0.000)$. The CT sensitivity was better only than PET/CT $(P=0.000)$ and was not inferior to CEUS $(P=1.000)$. In assessing CRCLM, the performance of PET/CT proved inferior to the other techniques, in sensitivity, although also in accuracy, in a lesion-by-lesion analysis.

In the group- $\mathrm{A}$, we observed statistically significant differences in sensitivity among all the methods (Cochran's $Q$ 18.783; $P=0.001)$. The sensitivity of i-CEUS, MR, CT, CEUS and PET/CT was $98 \%, 94 \%, 91 \%, 84 \%$ and $78 \%$, respectively. i-CEUS was statistically more sensitive only than PET $(P=0.000)$ and CEUS (0.016). In this group of upfront patients resected, the sensitivity of MR, CT and CEUS did not differ statistically. As was observed throughout the series, in this set of patients, too, PET/CT revealed significantly worse sensitivity than MR $(P=0.031)$, CT $(P=0.021)$ and i-CEUS $(P=0.000)$.

The $Q$-test was also statistically significant $(87.253 ; P=0.000)$ in group-B, consisting of lesions belonging to patients operated on after having received chemotherapy. The sensitivity of $\mathrm{PET} / \mathrm{CT}$ was $48 \%$, significantly lower when compared pairwise with all the other techniques $(P=0.000)$. i-CEUS proved equivalent in sensitivity to MR ( $95 \%$ and $90 \%$, respectively,

\begin{tabular}{|c|c|c|c|c|c|c|}
\hline & \multicolumn{2}{|c|}{ Total } & \multicolumn{2}{|c|}{ Group-A } & \multicolumn{2}{|c|}{ Group-B } \\
\hline & $n$ & $\%$ & $n$ & $\%$ & $n$ & $\%$ \\
\hline Total number of resected lesions & 175 & 100 & 67 & 38.3 & 108 & 61.7 \\
\hline \multicolumn{7}{|l|}{ Histology of resected lesions } \\
\hline Colorectal cancer liver metastasis & 155 & 88.6 & 59 & 88.1 & 96 & 88.9 \\
\hline No-colorectal cancer liver metastasis & 20 & 11.4 & 8 & 11.9 & 12 & 11.1 \\
\hline Hamartoma & 1 & 0.6 & 1 & 1.5 & 0 & 0 \\
\hline Cyst & 4 & 2.3 & 4 & 6 & 0 & 0 \\
\hline Necrosis & 7 & 4 & 0 & 0 & 7 & 6.5 \\
\hline Giant-cell reaction & 3 & 1.7 & 1 & 1.5 & 2 & 1.9 \\
\hline Focal nodular hyperplasia & 1 & 0.6 & 0 & 0 & 1 & 0.9 \\
\hline Steatosis & 4 & 2.3 & 0 & 0 & 4 & 3.7 \\
\hline \multicolumn{7}{|l|}{ Onset time of liver lesions } \\
\hline Synchronous & 133 & 76 & 40 & 59.7 & 93 & 86.1 \\
\hline Metachronous & 42 & 24 & 27 & 40.3 & 15 & 13.9 \\
\hline $\begin{array}{l}\text { Abbreviations: Group }-\mathrm{A}=\text { patients direct } \\
\text { preoperative chemotherapy. }\end{array}$ & & & & atie & & ed afte \\
\hline
\end{tabular}

$P=0.227)$ and both were significantly more sensitive than other procedures such as CT and PET/CT.

The sensitivity of all RITs was higher in group-A than in group-B (Figure 1). On other words, greater loss of sensitivity was observed for all techniques under examination in patients operated on after chemotherapy than was observed in patients treated directly with a surgical approach. The loss of sensitivity was statistically significant for CT $(91 \%$ vs $77 \% ; P=0.024)$ and PET/CT (78\% vs 48\%; $P=0.000)$ at the $\chi^{2}$-test. A similar situation was also observed for accuracy (Figure 2).

$\mathrm{PET} / \mathrm{CT}$ role in extrahepatic disease. We also analysed the impact of PET/CT in all 84 patients enrolled in the study who did not undergo radical surgery because of extrahepatic disease (Table 4). Eleven out of 14 patients with extrahepatic disease who performed both PET/CT and CT were evaluated. In these 11 patients, extraepatic disease was detected using CT and/or PET/CT or only by exploratory laparotomy. CT and PET/CT equally detected extraepatic disease in five patients: one abdominal lymph nodes, one bone, one abdominal lymph nodes/bone, one abdominal lymph nodes/lung and one lung/bone. In two cases, bone (one patient) and peritoneal carcinomatosis (one patient) were detected using PET/CT scan despite CT negativity. In four patients, CT and PET/CT failed to show extrahepatic disease but surgery would reveal peritoneal carcinomatosis (two patients) or inoperable abdominal lymph node metastases (two patients).

\section{DISCUSSION}

In the last two decades, there has been an increasingly more aggressive approach to surgery for LM, favoured by greater knowledge of liver anatomy and physiology, as well as by constant innovation in therapeutic options (Vigano et al, 2012). In selecting patients who may benefit from LM surgery it is essential to employ noninvasive RIT enabling correct assessment of the characteristics, number, distribution and size of hepatic lesions (Van Erkel et al, 2002; Fernandez et al, 2004; Rappeport et al, 2007). Several past studies have compared the different methods of imaging in a preoperative assessment of patients with CRCLM, but often the 


\begin{tabular}{|c|c|c|c|c|c|c|c|c|c|c|}
\hline & $\mathrm{CT}$ & $\%$ & MR & $\%$ & PET & $\%$ & CEUS & $\%$ & i-CEUS & $\%$ \\
\hline \multicolumn{11}{|l|}{ Sensitivity } \\
\hline All lesions & $127 / 154$ & 82 & $127 / 139$ & 91 & $85 / 141$ & 60 & $116 / 143$ & 81 & $146 / 152$ & 96 \\
\hline Group-A & $53 / 58$ & 91 & $44 / 47$ & 94 & $46 / 59$ & 78 & $48 / 57$ & 84 & $55 / 56$ & 98 \\
\hline Group-B & $74 / 96$ & 77 & $83 / 92$ & 90 & $39 / 82$ & 48 & $68 / 86$ & 79 & $91 / 96$ & 95 \\
\hline \multicolumn{11}{|c|}{ Positive predictive value } \\
\hline All lesions & $127 / 135$ & 94 & $127 / 134$ & 95 & $85 / 87$ & 98 & $116 / 126$ & 92 & $146 / 157$ & 93 \\
\hline Group-A & $53 / 54$ & 98 & $44 / 44$ & 100 & $46 / 47$ & 98 & $48 / 49$ & 98 & $55 / 57$ & 96 \\
\hline Group-B & $7 / 81$ & 91 & $83 / 90$ & 92 & $39 / 40$ & 98 & $68 / 77$ & 88 & $91 / 100$ & 91 \\
\hline \multicolumn{11}{|l|}{ Specificity } \\
\hline All lesions & $12 / 20$ & 60 & $10 / 17$ & 59 & $18 / 20$ & 90 & $10 / 20$ & 53 & $9 / 20$ & 45 \\
\hline Group-A & $7 / 8$ & 88 & $5 / 5$ & 100 & $7 / 8$ & 88 & $7 / 8$ & 88 & $6 / 8$ & 75 \\
\hline Group-B & $5 / 12$ & 42 & $5 / 12$ & 42 & $11 / 12$ & 92 & $3 / 12$ & 25 & $3 / 12$ & 25 \\
\hline \multicolumn{11}{|c|}{ Negative predictive value } \\
\hline All lesions & $12 / 39$ & 31 & $10 / 22$ & 45 & $18 / 74$ & 24 & $10 / 37$ & 27 & 9/15 & 60 \\
\hline Group-A & $7 / 12$ & 58 & $5 / 8$ & 63 & $7 / 20$ & 35 & $7 / 16$ & 44 & $6 / 7$ & 86 \\
\hline Group-B & $5 / 27$ & 19 & $5 / 14$ & 36 & $11 / 54$ & 20 & $3 / 21$ & 14 & $3 / 8$ & 38 \\
\hline \multicolumn{11}{|l|}{ Accuracy } \\
\hline All lesions & $139 / 174$ & 80 & $137 / 156$ & 88 & $103 / 161$ & 64 & $126 / 163$ & 77 & $155 / 172$ & 90 \\
\hline Group-A & $60 / 66$ & 91 & $49 / 52$ & 94 & $53 / 67$ & 79 & $55 / 65$ & 85 & $61 / 64$ & 95 \\
\hline Group-B & 79/108 & 73 & 89/104 & 85 & $50 / 94$ & 53 & $71 / 98$ & 72 & $94 / 108$ & 87 \\
\hline
\end{tabular}

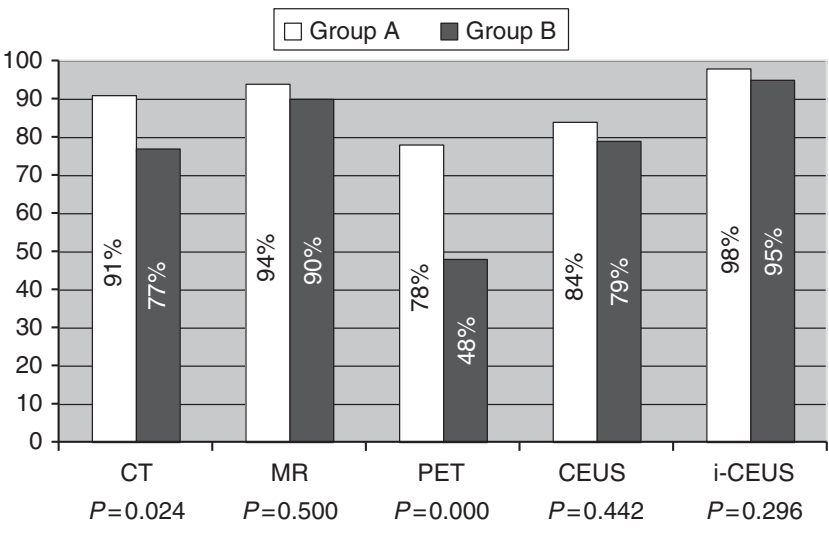

Figure 1. Lesion-by-lesion comparison of sensitivity in Group A and Group B.

reference standard used was heterogeneous and, moreover, the comparison involved different groups of patients (Huebner et al, 2000; Kinkel et al, 2002; Bipat et al, 2005; Rappeport et al, 2007; Rappeport and Loft, 2007).

The aim of the PROMETEO-01 study was to select the most suitable available RIT for planning surgery on patients with CRCLM, giving them a better chance of cure and hence survival. Lesion-by-lesion analysis in all patients showed that in the assessment of CRCLM, i-CEUS had the highest sensitivity, albeit not statistically better than MR $(96 \%$ vs $91 \% ; P=0.092)$. MR was more sensitive than CT (91\% vs $82 \% ; P=0.002)$, CEUS ( $91 \%$ vs $81 \% ; P=0.008)$ and PET/CT $(91 \%$ vs $60 \% ; P=0.000)$, whereas $\mathrm{PET} / \mathrm{CT}$ showed the lowest sensitivity. Moreover, CT sensitivity was not inferior to CEUS ( $82 \%$ vs $81 \% ; P=1.000)$. From our

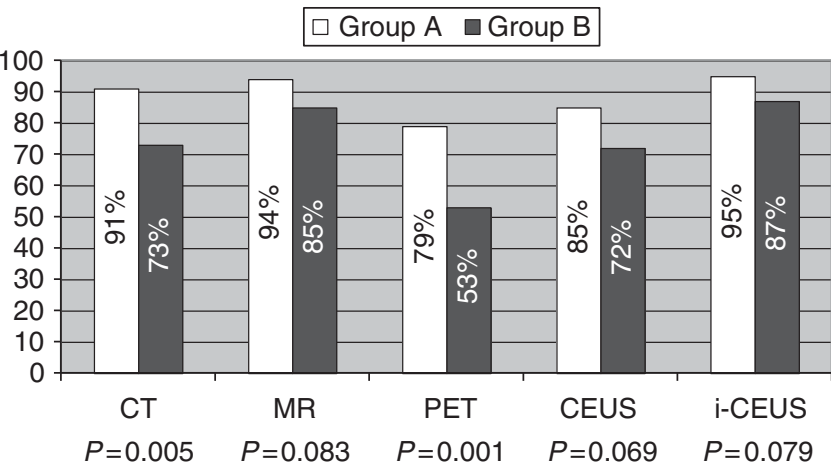

Figure 2. Lesion-by-lesion comparison of accuracy in Group A and Group B.

findings it could be concluded that in current clinical practice it may be sufficient to perform an i-CEUS or an MR in the pre-surgical assessment of CRCLM patients. However, we should consider that $\mathrm{i}$-CEUS is carried out during the operation, and does not therefore exclude a priori unresectable patients; on the other hand, an MR performed preoperatively would not detect extrahepatic disease. The CT with a sensitivity of $82 \%$ and an accuracy of $73 \%$ seems to be the most reasonable choice for staging patients towards surgery because it enables extrahepatic disease to be assessed while having a good likelihood of correctly mapping intrahepatic metastases.

When we evaluated Group-A, i-CEUS showed the highest sensitivity (94\%), although statistically it was only better than PET/CT $(P=0.000)$ and CEUS (0.016). Moreover, in this group of patients, PET/CT showed worse sensitivity than MR $(P=0.031)$, CT $(P=0.021)$ and i-CEUS $(P=0.000)$. As we found no 


\begin{tabular}{|c|c|c|c|c|}
\hline Patient & $\begin{array}{c}\text { Site of extrahepatic } \\
\text { disease }\end{array}$ & PET/CT & $\mathrm{CT}$ & Surgery \\
\hline MF & $\begin{array}{l}\text { Lung } \\
\text { Bone }\end{array}$ & $\begin{array}{l}+ \\
+\end{array}$ & $\begin{array}{l}+ \\
+\end{array}$ & \\
\hline $\mathrm{CL}$ & Peritoneum & + & - & \\
\hline$B C$ & Lymph nodes & - & + & \\
\hline SS & $\begin{array}{l}\text { Lymph nodes } \\
\text { Bone }\end{array}$ & $\begin{array}{l}+ \\
+\end{array}$ & $\begin{array}{l}+ \\
+\end{array}$ & \\
\hline WV & Peritoneum & - & - & + \\
\hline BA & Bone & + & + & \\
\hline SG & Lymph nodes & + & + & \\
\hline $\mathrm{BI}$ & Lymph nodes & - & - & + \\
\hline BA & $\begin{array}{c}\text { Lung } \\
\text { Lymph nodes }\end{array}$ & $\begin{array}{l}+ \\
+\end{array}$ & $\begin{array}{l}+ \\
+\end{array}$ & \\
\hline FR & Peritoneum & - & - & + \\
\hline TG & Bone & + & - & \\
\hline
\end{tabular}

significant differences between CT, CEUS and MR, we think the most appropriate choice in this population is CT, which allows complete staging of candidates for upfront surgery with acceptable sensitivity (91\%) and accuracy (91\%) in the detection of CRCLM.

In Group-B, i-CEUS shows equivalent sensitivity to MR (95\% and $90 \%$, respectively, $P=0.227$ ) and both are significantly more sensitive than other procedures such as CT and PET/CT. However, as an i-CEUS can only be performed during the operation, we believe the MR-which shows the best sensitivity (91\%) in detection of CRCLM in patients receiving chemotherapyremains the first choice test before surgery. CT sensitivity in this group of patients was significantly worse than in resectable patients $a b$ initio $(77 \%$ vs $91 \% ; P=0.024)$ and this may justify the restriction of its employment to the preliminary phase of staging, together with MR. The MR, on the other hand, should be repeated after chemotherapy in order to plan liver surgery appropriately.

In a prospective trial, Rappeport et al (2007) evaluated the diagnostic performance of PET/CT, PET, SPIO-enhanced MR and CT in the detection of CRCLM using surgery as the gold standard, whereas for benign lesions the gold standard was CT and/or MR findings. CT and SPIO-enhanced MRIs were significantly more sensitive $(P<0.0001)$ but less specific than PET $(66 \%)$ in the detection of LM. This contrasts with three previous meta-analyses that considered PET as the most sensitive non-invasive imaging for the detection of CRCLM (Huebner et al, 2000; Kinkel et al, 2002; Bipat et al, 2005). In the first meta-analysis, PET sensitivity in detecting LM was $96 \%$ and $91 \%$, respectively, per-patient and perlesion (Huebner et al, 2000).

Kinkel et al (2002) performed per-patient analysis to assess US, CT, MR and PET to detect LM from gastrointestinal tract tumours. The median sensitivity of PET was $90 \%$ and was significantly better than CT and MR. Bipat et al (2005) carried out both per-patient and per-lesion analyses in order to identify the sensitivity of CT, MR and PET. PET showed the best sensitivity in a per-patient analysis; however, when the same analysis was performed per-lesion, the PET sensitivity decreased significantly (from 94.6 to $75.9 \%$ ). This meta-analysis goes to show that in the strategy of surgical planning it is important for a diagnostic procedure to be able to provide the number, size and characteristics of LM, and this is best appraised by per-lesion analysis. This meta-analysis did not assess specificity in order to avoid the risk of underestimation

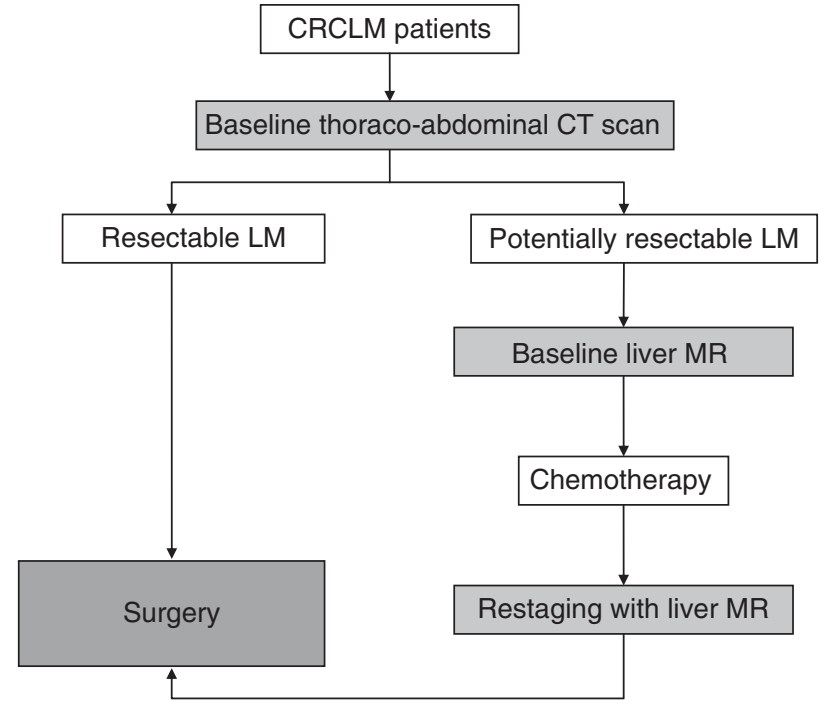

Figure 3. Summary of proposal for assessment in patients with CRCLM.

because the population under study was highly selected for LM. On the other hand, they do point out that specificity in an unselected population is likely to be overestimated. Their per-lesion analysis also showed the superiority of MR sensitivity over other methods.

The meta-analyses by Kinkel et al and Bipat et al compared imaging techniques that are now considered far from 'state of art'; they also included studies where the standard of reference was not always appropriate. In a prospective study, comparing two types of MR and CT (Gd. and SPIO) Ward et al (2005) found that the accuracy of MR was higher than CT.

In a review of the literature,Rappeport and Loft (2007) conclude that for diagnoses of CRCLM, MR and CT are more sensitive than PET, when the comparison is made within the same group of patients and taking surgery as the reference standard. In the preoperative evaluation, MR with a liver-specific contrast agent has a better ability to discriminate between small LM and cysts than does CT. In addition, PET allows one to identify extrahepatic disease that could rule out liver surgery.

A recent prospective study compared CEUS, MDCT, two types of MR (Gd-enhanced and SPIO-enhanced) and PET/CT in detecting CRCLM using as a reference standard bimanual palpation, IOUS, histology and follow-up CT on non-resected lesions. In the per-lesion analysis, Gd and SPIO-MRI were the most accurate in identifying CRCLM, whereas in per-patient analysis PET/CT shows an enhanced capacity for identifying LM (Mainenti et al, 2010).

In conclusion, despite the limitations related to the small related sample size, the PROMETEO-01 study results are in agreement with our clinical practice as currently guided by multidisciplinary discussion (the proposal flow chart is summarised in Figure 3). In our series the overall diagnostic accuracy of CT was $80 \%$ in all lesions, the extrahepatic disease was detected only by PET in $2.4 \%(2 / 84)$ of patients, the accuracy of RM was higher and potentially more useful in patients resected after chemotherapy. Therefore a thoraco-abdominal CT of good technical quality provides an adequate baseline evaluation and guides the resectability judgement of CRCLM patients. PET/CT is employed as a second-level examination to confirm any suspicion of extrahepatic disease. In the subset of patient candidates for induction chemotherapy to increase the chances of liver resection, the most rational approach is to add MR for the staging and restaging of liver disease. 


\section{REFERENCES}

Abdel-Misih SR, Schmidt CR, Bloomston PM (2009) Update and review of the multidisciplinary management of stage IV colorectal cancer with liver metastases. World J Surg Oncol 29: 7-72.

Bipat S, Van Leeuwen MS, Comans EF, Pijl ME, Bossuyt PM, Zwinderman AH, Stoker J (2005) Colorectal liver metastases: CT, MR imaging, and PET for diagnosis-meta-analysis. Radiology 237(1): 123-131.

Couinaud C (1957) Le foie: etudes anatomiques et chirurgicales. Masson: Paris.

Fernandez FG, Drebin JA, Linehan DC, Dehdashti F, Siegel BA, Strasberg SM (2004) Five-year survival after resection of hepatic metastases from colorectal cancer in patients screened by positron emission tomography with F-18 fluorodeoxyglucose (FDG-PET). Ann Surg 240: 438-447.

Huebner RH, Park KC, Shepherd JE, Schwimmer J, Czernin J, Phelps ME, Gambhir SS (2000) A meta-analysis of the literature for whole-body FDG PET detection of recurrent colorectal cancer. J Nucl Med 41(7): 1177-1189.

Isoniemi H, Osterlund P (2011) Surgery combined with oncological treatments in liver metastases from colorectal cancer. Scand J Surg 100: 35-41.

Kemeny N (2010) The management of resectable and unresectable liver metastases from colorectal cancer. Curr Opin Oncol 22(4): 364-373.

Kinkel K, Lu Y, Both M, Warren RS, Thoeni RF (2002) Detection of hepatic metastases from cancers of the gastrointestinal tract by using noninvasive imaging methods (US, CT, MR imaging, PET): a meta-analysis. Radiology 224(3): 748-756.

Lam VW, Spiro C, Laurence JM, Johnston E, Hollands MJ, Pleass HC, Richardson AJ (2012) A systematic review of clinical response and survival outcomes of downsizing systemic chemotherapy and rescue liver surgery in patients with initially unresectable colorectal liver metastases. Ann Surg Oncol 19(4): 1292-1301.

Mainenti PP, Mancini M, Mainolfi C, Camera L, Maurea S, Manchia A, Tanga M, Persico F, Addeo P, D'Antonio D, Speranza A, Bucci L, Persico G, Pace L, Salvatore M. (2010) Detection of colo-rectal liver metastases: prospective comparison of contrast enhanced US, multidetector CT, PET/CT, and 1.5 Tesla MR with extracellular and reticulo-endothelial cell specific contrast agents. Abdom Imaging 35(5): 511-521.

Masi G, Fornaro L, Caparello C, Falcone A (2011) Liver metastases from colorectal cancer: how to best complement medical treatment with surgical approaches. Future Oncol 7(11): 1299-1323.

Morris EJ, Forman D, Thomas JD, Quirke P, Taylor EF, Fairley L, Cottier B, Poston G (2010) Surgical management and outcomes of colorectal cancer liver metastases. Br J Surg 97: 1110-1118.

Muratore A, Zimmitti G, Ribero D, Mellano A, Langella S, Capussotti L (2010) Resection margin and recurrence-free survival after liver resection of colorectal metastases. Ann Surg Oncol 17: 1324-1329.

Nathan H, Bridges JF, Cosgrove DP, Diaz Jr LA, Laheru DA, Herman JM, Schulick RD, Edil BH, Wolfgang CL, Choti MA, Pawlik TM (2012) Treating patients with colon cancer liver metastasis: a nationwide analysis of therapeutic decision making. Ann Surg Oncol 19(12): 3668-3676.

Quan D, Gallinger S, Nhan C, Auer RA, Biagi JJ, Fletcher GG, Law CH, Moulton CA, Ruo L, Wei AC, McLeod RS. Surgical Oncology Program at Cancer Care Ontario (2012) The role of liver resection for colorectal cancer metastases in an era of multimodality treatment: A systematic review. Surgery 151(6): 860-870.

Rappeport ED, Loft A. (2007) Liver metastases from colorectal cancer: imaging with superparamagnetic iron oxide (SPIO)-enhanced MR imaging, computed tomography and positron emission tomography. Abdom Imaging 32: 624-634.

Rappeport ED, Loft A, Berthelsen AK, von der Recke P, Larsen PN, Mogensen AM, Wettergren A, Rasmussen A, Hillingsoe J, Kirkegaard P, Thomsen C (2007) Contrast-enhanced FDG-PET/CT vs. SPIO-enhanced MRI vs. FDG-PET vs. CT in patients with liver metastases from colorectal cancer: a prospective study with intraoperative confirmation. Acta Radiol 48(4): 369-378.

Ruers T, Bleichrodt RP (2002) Treatment of liver metastases, an update on the possibilities and results. Eur J Cancer 38: 1023-1033.

Sorbye H, Mauer M, Gruenberger T, Glimelius B, Poston GJ, Schlag PM, Rougier P, Bechstein WO, Primrose JN, Walpole ET, Finch-Jones M, Jaeck D, Mirza D, Parks RW, Collette L, Van Cutsem E, Scheithauer W, Lutz MP, Nordlinger B. EORTC Gastro-Intestinal Tract Cancer Group; Cancer Research UK (CRUK); Arbeitsgruppe Lebermetastasen undtumoren in der Chirurgischen Arbeitsgemeinschaft Onkologie (ALMCAO); Australasian Gastro-Intestinal Trials Group (AGITG); Fédération Francophone de Cancérologie Digestive (FFCD) (2012) Predictive factors for the benefit of perioperative FOLFOX for resectable liver metastasis in colorectal cancer patients (EORTC Intergroup Trial 40983). Ann Surg 255(3): 534-539.

Torzilli G (2004) Contrast-enhanced intraoperative ultrasonography in surgery for liver tumors. Eur J Radiol 51: S25-S29.

Van Cutsem E, Nordlinger B, Adam R, Köhne CH, Pozzo C, Poston G, Ychou M, Rougier P. European Colorectal Metastases Treatment Group (2006) Towards a pan-European consensus on the treatment of patients with colorectal liver metastases. Eur J Cancer 42: 2212-2221.

Van Erkel AR, Pij ME, Van Den Berg-Huysmans AA, Wasser MN, van de Velde CJ, Bloem JL (2002) Hepatic metastases in patients with colorectal cancer: relationship between size of metastases, standard of reference, and detection rates. Radiology 224(2): 404-409.

Vigano L, Russolillo N, Ferrero A, Langella S, Sperti E, Capussotti L (2012) Evolution of long-term outcome of liver resection for colorectal metastases: analysis of actual 5-year survival rates over two decades. Ann Surg Oncol 19: 2035-2044.

Wagner JS, Adson MA, VanHerder JA, Adson MH, Ilstrup DM (1984) The natural history of hepatic metastases from colorectal cancer. Ann Surg 199(5): 502-507.

Ward J, Robinson PJ, Guthrie JA, Downing S, Wilson D, Lodge JP, Prasad KR, Toogood GJ, Wyatt JI (2005) Liver metastases in candidates for hepatic resection: comparison of helical CT and gadolinium- and SPIO-enhanced MR imaging. Radiology 237(1): 170-180.

This work is published under the standard license to publish agreement. After 12 months the work will become freely available and the license terms will switch to a Creative Commons AttributionNonCommercial-Share Alike 3.0 Unported License. 